\title{
Extent of Surgical Resection in Lower-Grade Gliomas: Differential Impact Based on Molecular Subtype
}

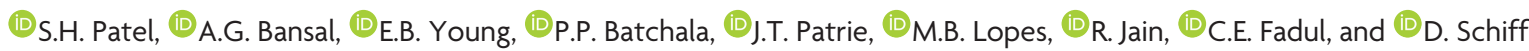

\begin{abstract}
BACKGROUND AND PURPOSE: Diffuse lower-grade gliomas are classified into prognostically meaningful molecular subtypes. We aimed to determine the impact of surgical resection on overall survival in lower-grade glioma molecular subtypes.
\end{abstract}

MATERIALS AND METHODS: For 172 patients with lower-grade gliomas (World Health Organization grade II or III), pre- and postsurgical glioma volumes were determined using a semiautomated segmentation software based on FLAIR or T2-weighted MR imaging sequences. The association of pre- and postsurgical glioma volume and the percentage of glioma resection with overall survival was determined for the entire cohort and separately for lower-grade glioma molecular subtypes based on isocitrate dehydrogenase (IDH) and lp/19q status, after adjustment for age, sex, World Health Organization grade, chemotherapy administration, and radiation therapy administration.

RESULTS: For the entire cohort, postsurgical glioma volume (hazard ratio, 1.80; 95\% Cl, 1.18-2.75; $P=.006$ ) and the percentage of resection (hazard ratio, 3.22; 95\% Cl, 1.79-5.82; $P<.001$ ) were associated with overall survival. For IDH-mutant 1p/19q-codeleted oligodendrogliomas, the percentage of resection (hazard ratio, $6.69 ; 95 \% \mathrm{Cl}, 1.57-28.46 ; P=.01$ ) was associated with overall survival. For IDH-mutant 1p/19q-noncodeleted astrocytomas, presurgical glioma volume (hazard ratio, 3.20; $95 \% \mathrm{Cl}, 1.22-8.39 ; \mathrm{P}=.018$ ), postsurgical glioma volume (hazard ratio, $2.33 ; 95 \% \mathrm{Cl}$, $1.32-4.12 ; P=.004$ ), and percentage of resection (hazard ratio, $4.34 ; 95 \% \mathrm{Cl}, 1.74-10.81 ; P=.002$ ) were associated with overall survival. For $\mathrm{IDH}$-wild-type lower-grade gliomas, pre-/postsurgical glioma volume and percentage of resection were not associated with overall survival.

CONCLUSIONS: The extent of surgical resection has a differential survival impact in patients with lower-grade gliomas based on their molecular subtype. IDH-mutant lower-grade gliomas benefit from a greater extent of surgical resection, with the strongest impact observed for IDH-mutant 1p/19q-noncodeleted astrocytomas.

ABBREVIATIONS: LGG = lower-grade glioma; mut = mutation; WHO = World Health Organization; IDHmut-Noncodel $=$ LGGs with an IDH mutation but lacking Ip/19q codeletion; IDHmut-Codel = LGGs with an IDH mutation and codeletion of chromosome arms Ip and 19q; IDHwt = LGGs in IDH-wild-type subgroup

D iffuse lower-grade gliomas (LGGs) are World Health Organization (WHO) grade II and III infiltrative brain neoplasms. In 2016, the WHO updated the classification of diffuse LGGs by integrating molecular markers with histopathology. ${ }^{1} \mathrm{Be}-$ tween $70 \%$ and $80 \%$ of LGGs are now known to have a prognostically favorable mutation in the isocitrate dehydrogenase (IDH) gene. ${ }^{2,3}$ Oligodendrogliomas (grade II) and anaplastic oligoden-

Received March 27, 2019; accepted after revision May 12.

From the Departments of Radiology and Medical Imaging (S.H.P., A.G.B., E.B.Y., P.P.B.), Public Health Sciences (J.T.P.), and Pathology (M.B.L.), Divisions of Neuropathology and Molecular Diagnostics, and Division of Neuro-Oncology (C.E.F., D.S.), University of Virginia Health System, Charlottesville, Virginia; and Departments of Radiology (R.J.) and Neurosurgery (R.J.), New York University School of Medicine, New York, New York.

S.H. Patel received a Radiological Society of North America Research Scholar Grant (RSCH1819).

Paper previously presented at: American Society of Neuroradiology Annual Meeting and the Foundation of the ASNR Symposium, May 18-23, 2019; Boston, Massachusetts. drogliomas (grade III) are defined by the presence of both an $I D H$ mutation and codeletion of chromosome arms $1 \mathrm{p}$ and $19 \mathrm{q}$ (heretofore collectively referred to as IDHmut-Codel). LGGs with an IDH mutation but lacking $1 \mathrm{p} / 19 \mathrm{q}$ codeletion include diffuse astrocytomas (grade II) and anaplastic astrocytomas (grade III) (heretofore collectively referred to as IDHmut-Noncodel). ${ }^{1,3-5}$ These IDH-mutant LGG subgroups have distinct prognostic and therapeutic profiles. $^{3-7}$ The IDH-wild-type subgroup of LGGs (heretofore collectively referred to as $I D H w t$ ) is associated with the most aggressive clinical behavior and worst outcome, similar to that of glioblastomas (WHO grade IV), though heterogeneity in the behavior and molecular features within this subgroup is being increasingly recognized. ${ }^{8-11}$

Please address correspondence to Sohil H. Patel, MD, Department of Radiology and Medical Imaging, University of Virginia Health System, PO Box 800170, Charlottesville, VA 22908; e-mail: shp4k@virginia.edu

Indicates article with supplemental on-line photo.

http://dx.doi.org/10.3174/ajnr.A6102

AJNR Am J Neuroradiol 40:1149-55 Jul 2019 www.ajnr.org 
Multiple studies have provided evidence supporting the maximum safe surgical resection as the front-line treatment of diffuse LGGs. ${ }^{12-19}$ However, most studies were undertaken before the 2016 WHO update for the classification of diffuse LGGs, without accounting for defining molecular markers. It is unknown to what extent these earlier study results are confounded by the use of older nonmolecular classification schema. Only recently have volumetric MR imaging studies incorporated $I D H$ and $1 \mathrm{p} / 19 \mathrm{q}$-codeletion status when assessing the impact of surgical resection on patient outcomes in LGGs. ${ }^{20,21}$

The purpose of our study was to determine the effect of surgical resection extent on overall survival in a patient cohort with LGGs stratified by molecular subtype. Among the IDH-mutant LGGs, we hypothesized that the extent of surgery might have greater impact among IDHmut-Noncodel subtypes than the IDHmut-Codel subtypes, given the overall worse prognosis among the former subtype. We hypothesized that surgery would have prognostic impact among the IDHwt subtypes as well, though these results might be confounded by the molecular heterogeneity of this subtype.

\section{MATERIALS AND METHODS}

This retrospective study of patient data was Health Insurance Portability and Accountability Act-compliant and was approved by our institutional review board (University of Virginia Health System).

\section{Patient Selection}

The patients included in this study were selected from an institutional neuro-oncology/neuroradiology diffuse glioma data base maintained at our institution, containing a total of 429 diffuse glioma cases diagnosed between 2000 and 2018. All patients who are seen by Medical Neuro-Oncology at our institution are added to our data base. From this data base, patients were selected on the basis of the following inclusion criteria: 1) diagnosed with a diffuse LGG (WHO grades II and III), 2) a known molecular subtype based on IDH and 1p/19q-codeletion status, 3) available presurgical and postsurgical MR images, and 4) known chemotherapy and radiation therapy history at the last follow-up. After review of the electronic medical record and PACS, we excluded 176 cases with grade IV histology (ie, glioblastomas) and 49 LGGs with unknown molecular status. Of the remaining 204 cases, we excluded 20 cases for lack of pre- or postsurgical MR imaging and 12 cases for unknown chemotherapy or radiation therapy history at time of the last follow-up. A total of 172 patients met the criteria for study inclusion. The mean time interval between the presurgical MR imaging and the operation was 5.18 days, and the mean time interval between the postsurgical MR imaging and the operation was 79.3 days. As per the methodology of Wijnenga et al, ${ }^{20}$ we preferentially avoided using the immediate postsurgical MR imaging scans for performing volume measurements to avoid including postsurgical edema or ischemia in our measurements.

\section{Neuroimaging Analysis}

Pre- and postsurgical MR images were analyzed by a board-certified neuroradiologist with a Certificate of Added Qualification in diagnostic neuroradiology and 6 years of experience. Measure-
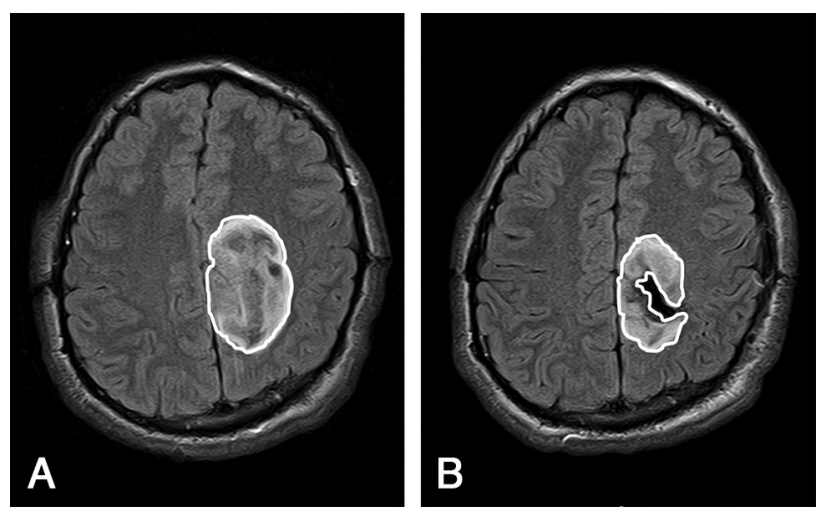

FIG 1. Glioma segmentation shown on axial FLAIR images of presurgical MR imaging $(A)$ and postsurgical MR imaging $(B)$ in a patient with a diffuse $I D H$-mutant astrocytoma who underwent subtotal resection.

ments of glioma volume on the presurgical and postsurgical MR imaging examinations were undertaken using the semiautomated lesion-management tool in the PACS using FLAIR or T2weighted imaging ( $3 \mathrm{D}$ sequences were used when possible). MR imaging examinations analyzed in this study were performed on various $1.5 \mathrm{~T}$ and $3 \mathrm{~T}$ MR imaging scanners, and pulse sequences with variable parameters were used. On 3T MR imaging, the 3DT2WI and FLAIR sequences used the following parameters-3DT2WI: FOV $=256 \mathrm{~mm}$, slice thickness $=1.0 \mathrm{~mm}$, matrix $=256 \times$ 256, $\mathrm{TR}=3200 \mathrm{~ms}, \mathrm{TE}=413 \mathrm{~ms}, \mathrm{NEX}=1 ; 3 \mathrm{D}$-FLAIR: $\mathrm{FOV}=$ $256 \mathrm{~mm}$, slice thickness $=1.0 \mathrm{~mm}$, matrix $=256 \times 256$, $\mathrm{TR}=$ $5000 \mathrm{~ms}, \mathrm{TI}=1800 \mathrm{~ms}, \mathrm{TE}=386 \mathrm{~ms}, \mathrm{NEX}=1$. To create glioma volumes of interest, the neuroradiologist reader manually traced the contour of the glioma using axial images from the highest quality FLAIR or T2-weighted sequence on the presurgical and postsurgical MR images (Fig 1), and the software calculated a 3D volume based on the neuroradiologist's segmentation. For the presurgical volume measurements, in cases in which peritumoral edema could not be confidently distinguished from infiltrative glioma, the neuroradiologist reader erred on the side of including any high signal present on the T2/FLAIR sequences within the volume measurements. Based on presurgical and postsurgical glioma volumes, the percentage of glioma resection was calculated as $100 \% \times(1-$ [Postsurgical Glioma Volume] / [Presurgical Glioma Volume]).

\section{Neuropathology}

Glioma histology and grade and $I D H$ and $1 \mathrm{p} / 19 \mathrm{q}$ statuses were retrieved from the electronic medical record. $I D H$ and $1 \mathrm{p} / 19$ statuses were tested in the Clinical Laboratory Improvement Amendments-certified molecular pathology laboratory at our institution. $I D H$-mutation status was first tested by immunohistochemistry using an IDH1 R132H-mutant-specific antibody. Immunohistochemistry was performed on 4 - $\mu$ m-thick sections from formalin-fixed paraffin-embedded tissue following the manufacturer's recommended protocol (Bond-III; Leica Biosystem, Newcastle Upon Tyne, UK). Commercially purchased antibodies against IDH1 $(\mathrm{R} 132 \mathrm{H})$ mutant protein (DIA-H09; Dianova, Hamburg, Germany) were used. ${ }^{22,23}$ In cases with negative findings on immunohistochemistry, $I D H 1 / 2$ 
Table 1: Summary of patient characteristics ${ }^{a}$

\begin{tabular}{lccc}
\hline & $\begin{array}{c}\text { IDHmut-Codel } \\
(\boldsymbol{n}=68)\end{array}$ & $\begin{array}{c}\text { IDHmut-Noncodel } \\
(\boldsymbol{n}=63)\end{array}$ & $\begin{array}{c}\text { IDHwt } \\
(\boldsymbol{n}=41)\end{array}$ \\
\hline Sex & $37(54.4 \%)$ & $30(47.6 \%)$ & $18(43.9 \%)$ \\
$\quad$ Female & $31(45.6 \%)$ & $33(52.4 \%)$ & $23(56.1 \%)$ \\
$\quad$ Male & $45.5(35.5-53)$ & $33(27-42.8)$ & $57.0(49.5-63.3)$ \\
Age (yr) & & & \\
WHO grade & $50(73.5 \%)$ & $46(73.0 \%)$ & $25(61.0 \%)$ \\
$\quad$ II & $18(26.5 \%)$ & $17(27.0 \%)$ & $16(39.0 \%)$ \\
III & & & \\
Chemotherapy & $55(80.9 \%)$ & $53(84.1 \%)$ & $30(73.2 \%)$ \\
$\quad$ Yes & $13(19.1 \%)$ & $10(15.9 \%)$ & $11(26.8 \%)$ \\
$\quad$ No & $45(66.2 \%)$ & $53(84.1 \%)$ & $31(75.6 \%)$ \\
Radiation therapy & $23(33.8 \%)$ & $10(15.9 \%)$ & $10(24.4 \%)$ \\
$\quad$ Yes & $56.4(26.5-111.6)$ & $55.0(31.0-116.2)$ & $47.7(18.0-86.4)$ \\
$\quad$ No & $12(17.6 \%)$ & $14(22.2 \%)$ & $30.1(8.4-64.4)$ \\
Presurgical glioma volume $\left(\mathrm{cm}^{3}\right)^{\mathrm{a}}$ & $13(31.7 \%)$ \\
\hline $\begin{array}{l}\text { Postsurgical glioma volume }\left(\mathrm{cm}^{3}\right)^{\mathrm{a}} \\
\text { Mortality events }\end{array}$ & $28.4(13.2-59.1)$ & $29.3(1.2-65.6)$ & \\
\hline
\end{tabular}

${ }^{2}$ Data are listed as median and interquartile range. All other data are listed as absolute values and percentages.

(IDHmut-Codel, IDHmut-Noncodel, ID$H w t$, or all molecular subtypes combined). Per variable of interest (eg, presurgical glioma volume) and per molecular subtype of patients (eg, the IDHmut-Noncodel molecular subtype), the regression model included not only the variable of interest (eg, presurgical glioma volume) but also patient age and sex, WHO grades II and III, chemotherapy administration status (yes, no), and radiation therapy administration status (yes, no) as concomitant variable predictors of survival time. For all 12 multivariate Cox models, the survival times of patients who were known to be alive at last follow-up were treated as rightcensored observations. With regard to hypothesis testing, the type III version of

mutation status was assessed by the clinically validated DNA pyrosequencing assay, using the PyroMark Q24 system, following the manufacturer's recommended protocol (QIAGEN; https://www.qiagen.com/us/products/discovery-andtranslational-research/pyrosequencing/instruments/pyromarkq24/\#orderinginformation).

We used the following primers-IDH1 forward primer: 5'-Biot.-CATAATGTTGGCGTCAAATGTG-3'; IDH1 reverse primer: 5' -ACATGCAAAATCACATTATTGCC-3'; IDH1 sequencing primer: 5'-TGATCCCCATAAGCAT-3'; IDH2 forward primer: 5'-GTTCAAGCTGAAGAAGATGTGG-3'; IDH2 reverse primer: 5'-Biot.-GTGGCCTTGTACTGCAGAG-3'; IDH2 sequencing primer: and 5'-AGCCCATCACCATTGG-3'. The pyrosequencing assay is designed to detect mutations within codon 132 of IDH1 and codon 172 of $I D H 2$, as described previously. ${ }^{24}$ The $1 \mathrm{p} / 19 \mathrm{q}$-codeletion status was determined using dual-color fluorescence in situ hybridization on formalin-fixed paraffin-embedded tissue. Commercial human probes were applied to localize 1p36, 1q25, 19p13 (Vysis; Abbott Molecular, Abbott Park, Illinois), and DAPI (Insitus Biotechnologies, Albuquerque, New Mexico) was used as a nuclear counterstain.

\section{Statistical Analysis}

Categoric variables are summarized by frequencies and percentages, and molecular subtype comparisons of categoric variables were conducted using the Fisher exact test. Continuous scale variables are summarized by the median, interquartile range, and range of the distribution, and molecular subtype comparisons of continuous scale variables were performed using the Wilcoxon rank sum test. Multivariate Cox proportional hazards regression was used to examine whether presurgical glioma volume, postsurgical glioma volume, and the percentage of glioma resection are uniquely associated with overall survival time for all cases and for cases in the 3 molecular subtypes. In total, 12 multivariate Cox models were constructed, and each model was identically specified with the only between-model difference being the variable of interest (presurgical glioma volume, postsurgical volume, or percentage of glioma resection) and the molecular subtype the Wald $\chi^{2}$ statistic served as the pivotal quantity for testing the null hypothesis that there is no unique association between the variable of interest (eg, presurgical glioma volume) and survival time after accounting for patient age and sex, WHO grade, chemotherapy administration status, and radiation therapy administration status associations with survival time. A $P \leq .05$ decision rule was established a priori as the null hypothesis rejection rule for testing for predictor variable-versus-survival time unique association, and the strength of the predictor variable versus survival time unique association was quantified by the adjusted hazard ratio.

\section{RESULTS}

Among the 172 study patients, there were 85 females (49.4\%) and 87 males (50.6\%). The median age was 43 years (interquartile range, $31-54$ years; range, $17-76$ years). Patient characteristics per molecular subtype are shown in Table 1 . There were significant differences in age in each of the 3 molecular subgroups $(P \leq .001)$, with patients with IDHwt being the oldest and those with IDHmut-Noncodel being the youngest. There were a total of 39 deaths. Median overall survival time was 16.5 years among patients with IDHmut-Codel, 12.1 years among those with IDHmutNoncodel, and 6.3 years among those with IDHwt. Overall survival curves for the LGG subtypes are shown in Fig 2. Overall survival curves differed between patients with IDHmut-Codel and IDHwt $(P<.001)$ and between those with IDHmut-Noncodel and IDHwt $(P<.001)$. Overall survival curves did not significantly differ between patients with IDHmut-Codel and IDHmut-Noncodel $(P=.125)$.

Among all cases, the median presurgical glioma volume was $54.7 \mathrm{~cm}^{3}$ (interquartile range, $27.6-107.6 \mathrm{~cm}^{3}$; range, $1.1-340$ $\mathrm{cm}^{3}$ ) and the median postsurgical glioma volume was $29.7 \mathrm{~cm}^{3}$ (interquartile range, $6.5-62.8 \mathrm{~cm}^{3}$; range, $0-339 \mathrm{~cm}^{3}$ ). There was no significant difference in presurgical glioma volume or postsurgical glioma volume among the 3 LGG molecular subtypes. Patients with IDHwt were associated with a smaller percentage of resection compared with those with IDHmut-Noncodel $(P=.01)$.

Associations among presurgical glioma volume, postsurgical

AJNR Am J Neuroradiol 40:1149-55 Jul 2019 www.ajnr.org 1151 


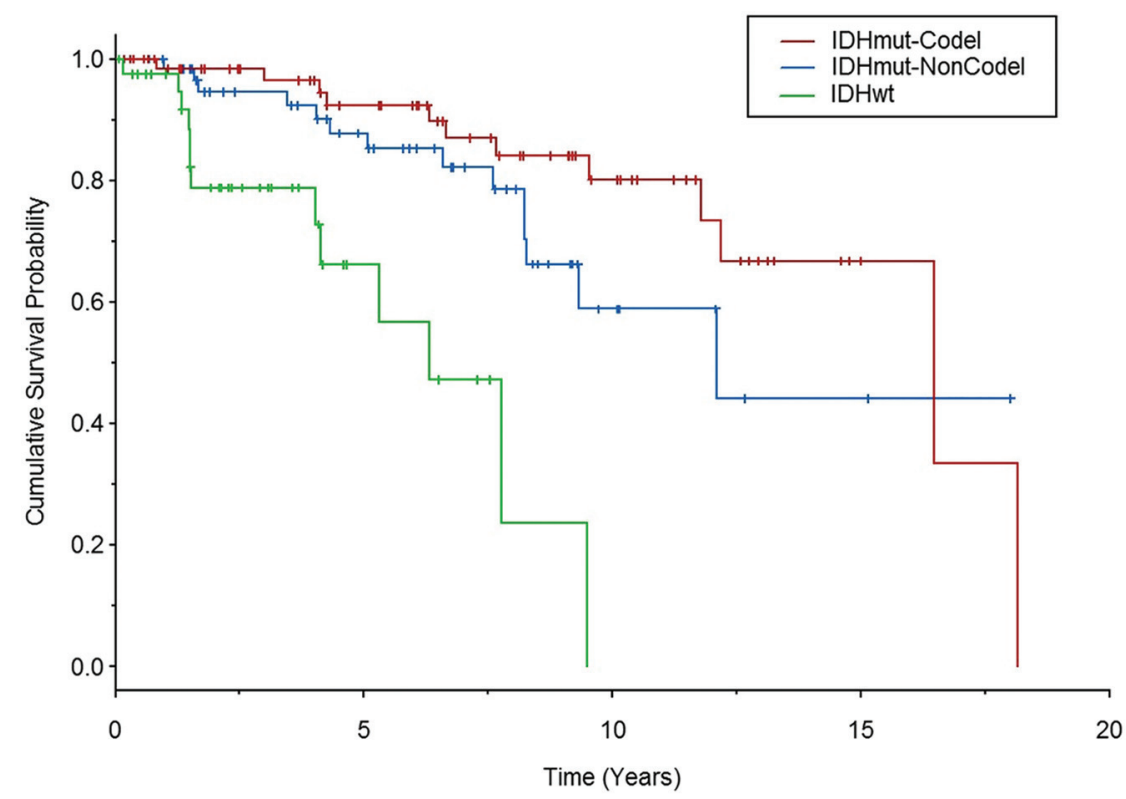

FIG 2. Kaplan-Meier curves for overall patient survival based on the LGG molecular subtype. Vertical line segments along the curve identify right-censored survival times. IDHwt LGGs were associated with significantly worse overall survival compared with both $I D H$-mutant subtypes.

Table 2: Survival analysis ${ }^{\mathrm{a}}$

\begin{tabular}{|c|c|c|c|}
\hline Predictor & Ratio & $\begin{array}{c}\text { Adjusted Hazard } \\
\text { Ratio }(95 \% \mathrm{Cl})\end{array}$ & $P$ Value \\
\hline \multicolumn{4}{|l|}{ All LGGs } \\
\hline Presurgical glioma volume & 3rd Q:1st Q & $1.14(0.79-1.64)$ & .479 \\
\hline Postsurgical glioma volume & 3rd Q:1st Q & $1.80(1.18-2.75)$ & .006 \\
\hline Percentage glioma resection & $25 \%: 75 \%$ & $3.22(1.79-5.82)$ & $<.001$ \\
\hline \multicolumn{4}{|l|}{ IDHmut-Codel } \\
\hline Presurgical glioma volume & 3rd Q:1st Q & $1.11(0.53-2.33)$ & .781 \\
\hline Postsurgical glioma volume & 3rd Q:1st Q & $1.67(0.82-3.48)$ & .170 \\
\hline Percentage glioma resection & $25 \%: 75 \%$ & $6.69(1.57-28.46)$ & .010 \\
\hline \multicolumn{4}{|l|}{ IDHmut-Noncodel } \\
\hline Presurgical glioma volume & 3rd Q:1st Q & $3.20(1.22-8.39)$ & .018 \\
\hline Postsurgical glioma volume & 3rd Q:1st Q & $2.33(1.32-4.12)$ & .004 \\
\hline Percentage glioma resection & $25 \%: 75 \%$ & $4.34(1.74-10.81)$ & .002 \\
\hline \multicolumn{4}{|l|}{ IDHwt } \\
\hline Presurgical glioma volume & 3rd Q:1st Q & $2.18(0.47-10.15)$ & .319 \\
\hline Postsurgical glioma volume & 3rd Q:lst Q & $1.34(0.28-4.76)$ & .653 \\
\hline Percentage glioma resection & $25 \%: 75 \%$ & $0.91(0.29-2.82)$ & .874 \\
\hline
\end{tabular}

Note:-Q indicates quartile.

a Predictor variables for overall survival are presurgical glioma volume, postsurgical glioma volume, and percentage glioma resection. Adjusted hazard ratios are listed for the entire cohort and for the 3 molecular subtypes, with age, sex, glioma grade, and chemotherapy and radiation therapy administration as the adjustment variables. percentage of glioma resection with overall survival. Figure 3 shows overall survival curves according to the percentage of glioma resection for each of the molecular subtypes, and the On-line Figure shows overall survival curves according to postsurgical glioma volume strata. There were 13 patients with $0 \mathrm{~mL}$ of postsurgical residual glioma (IDHmut-Codel, $n=1$; IDHmut-Noncodel, $n=9$; IDHwt, $n=3$ ), and all these patients were alive at the time of last follow-up.

\section{DISCUSSION}

Our study supports an association between a greater degree of surgical resection and overall survival in patients with $I D H$-mutant LGGs. We note an association between the extent of surgical resection and overall survival among both molecularly defined IDH-mutant astrocytomas and oligodendrogliomas; however, the impact of surgical resection extent on overall survival appears stronger for astrocytomas. The reason for the relatively stronger association with astrocytomas might relate to the relatively better responsiveness to nonsurgical therapies for oligodendrogliomas or oligodendrogliomas generally having longer survival times than astrocytomas, thus making it more difficult to demonstrate a survival benefit with an operation. ${ }^{3-7}$ We further note that the percentage of glioma resection was more strongly associated with overall survival than presurgical glioma volume or postsurgical glioma volume. Finally, we found no association between surgical resection and overall survival in $I D H w t$ LGGs.

Prior studies have investigated the impact of surgical resection on the outglioma volume, and the percentage of glioma resection and overall survival are shown in Table 2, after adjustment for patient age, sex, glioma grade, and chemo-radiation therapy administration. Among all LGGs in the cohort, postsurgical glioma volume ( $P=$ $.006)$ and the percentage of glioma resection $(P<.001)$ were associated with overall survival. However, associations varied among the 3 LGG molecular subtypes. For those with IDHmutCodel, the percentage of resection was associated with overall sur$\operatorname{vival}(P=.01)$, but pre- or postsurgical glioma volumes were not. For those with IDHmut-Noncodel, presurgical glioma volume $(P=.018)$, postsurgical glioma volume $(P=.004)$, and the percentage of glioma resection $(P=.002)$ were each associated with overall survival. For the IDHwt subtype, there was no association of presurgical glioma volume, postsurgical glioma volume, or comes of patients with LGGs. Among the older studies, many used nonvolumetric approximations of residual glioma extent. ${ }^{15,25-27}$ Additionally, most prior studies were undertaken before the 2016 WHO update for CNS neoplasm classification and thus did not strictly classify their patient cohorts by $I D H$ and $1 \mathrm{p} / 19 \mathrm{q}$-codeletion status. ${ }^{13,14,16}$ Only recently have studies provided evidence of the impact of surgical resection after stratification by defining molecular markers. ${ }^{20,21,28,29}$

The most important of this recent literature is the work from Wijnenga et al, in 2018, ${ }^{20}$ who evaluated the impact of surgery in a large cohort of molecularly defined grade II diffuse gliomas, determining that larger postoperative glioma volume was associated with worse overall survival (hazard ratio, 1.01 per $1 \mathrm{~cm}^{3}$ increase in residual glioma volume). They noted that the amount 

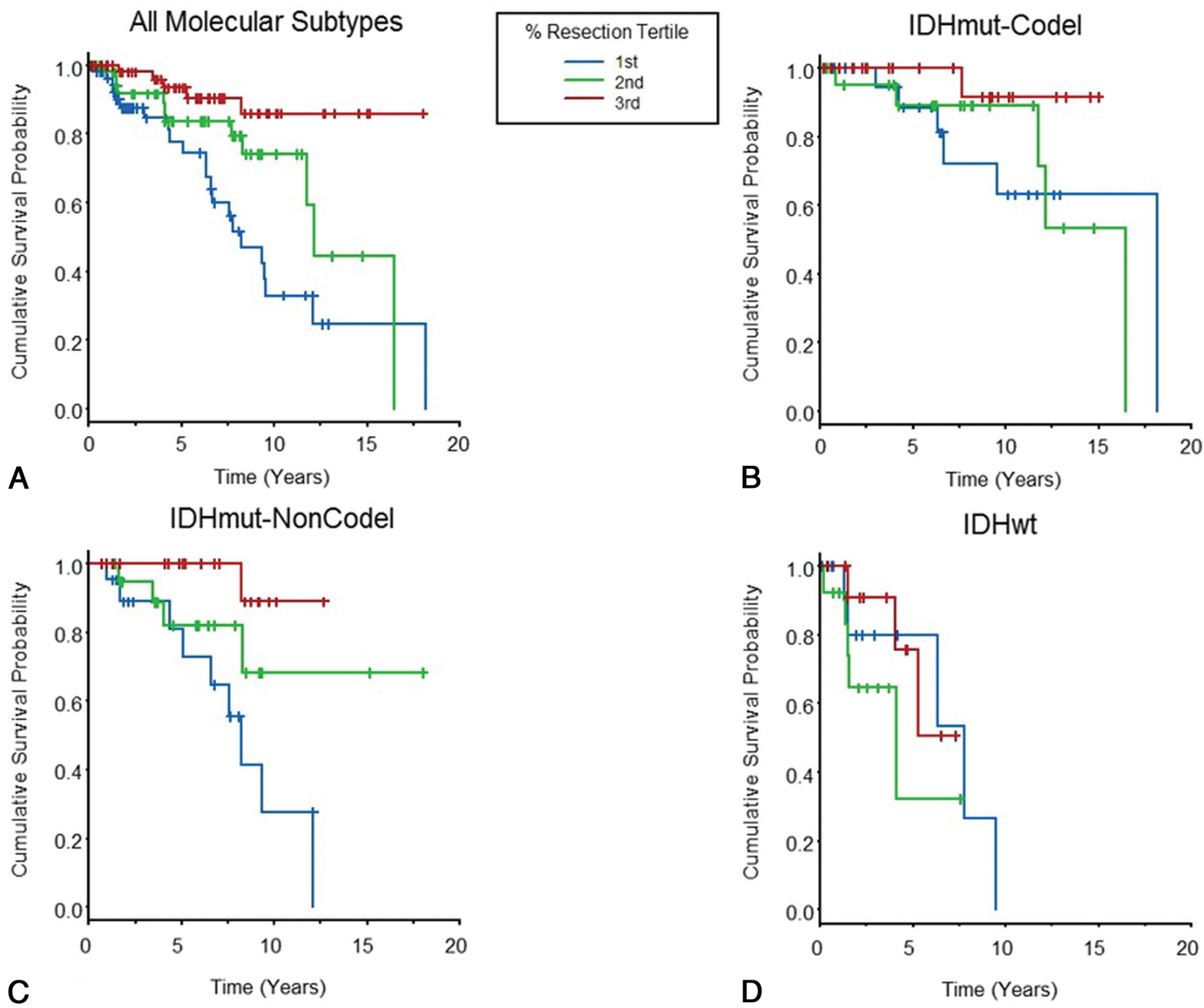

FIG 3. Kaplan-Meier curves in which overall patient survival is evaluated according to the percentage of glioma resection tertiles. Vertical line segments along the curve identify right-censored survival times. Data are shown for all patients with LGGs in the cohort $(A)$ and separately for the 3 molecular subtypes $(B-D)$.

of residual postsurgical glioma was more strongly associated with survival among IDH-mutant astrocytomas compared with oligodendrogliomas, similar to our results. Kawaguchi et al, in 2016, ${ }^{28}$ evaluated a cohort of WHO grade III diffuse gliomas, undertaking an impressively comprehensive molecular analysis of their glioma specimens given the timing of their study. They found that gross total resection (as determined by postoperative MR imaging analysis) was significantly associated with improved overall survival among the IDHmut-Noncodel subgroup, but not for the IDHmut-Codel or the IDHwt subgroup. Study limitations included their binary stratification of surgical resection (yes/no gross total resection) and their choice of measuring residual glioma differently for enhancing and nonenhancing gliomas.

Eseonu et al, in 2017, ${ }^{21}$ evaluated a cohort of low-grade (presumably WHO grade II) diffuse gliomas. Molecular information was only available in a 51-patient subset of the patient cohort, among whom the extent of resection was associated with improved survival for the codeleted oligodendroglioma subgroup and $I D H$-wild-type subgroup (they did not have sufficient $I D H$ - mutant astrocytomas for analysis). Patel et al, in 2018, ${ }^{29}$ evaluated a cohort of 74 patients with WHO grade II diffuse gliomas and, most interesting, found that the extent of glioma resection was associated with overall survival for the $I D H$-wild-type subgroup but not for the $I D H$-mutant subgroup, differing from our findings. Study limitations included lack of a description of $I D H$ mutation testing (with apparent lack of IDH2-mutation testing) and lack of stratification by $1 \mathrm{p} / 19 \mathrm{q}$-codeletion status.

The primary advantages of our study design include strict molecular classification of our patients with LGG, consistent with WHO guidelines, as well as volumetric measurement of pre-and postsurgical gliomas. Unlike most prior studies, we included both WHO grade II and III LGGs because WHO grade assignment among LGGs is subject to sampling bias and known to have significant interobserver variability. ${ }^{30}$ Moreover, we ensured that our statistical analysis controlled for tumor grade. We also controlled for additional potentially confounding variables such as patient age, sex, and chemo-radiation therapy administration by the time of last follow-up. Our finding that the extent of surgical resection impacts overall survival in patients with $I D H$-mutant 
LGGs, particularly in patients with IDHmut-Noncodel LGGs, supports most of the recent literature. Our finding that surgical resection does not impact survival in IDHwt LGGs is not broadly supported in the literature and requires further study. We speculate that disparate results regarding IDHwt LGGs may relate to the known clinical and molecular heterogeneity of this subgroup, especially among grade II IDHwt LGGs, ${ }^{8-10}$ and further stratification of this subgroup may be forthcoming in future WHO classification schemes. ${ }^{11}$

Our study has limitations. Its retrospective design may have introduced unanticipated biases that affected the results. Our analysis might be limited by our cohort size and follow-up time, given the number of mortality events. For example, our relatively smaller cohort of IDHwt LGGs might have impacted our ability to discern survival benefits from surgical resection in this subgroup. Moreover, although we adhered to the latest WHO criteria, we did not include more detailed molecular testing of IDHwt LGGs (eg, TERT promoter mutation, chromosome 7/10 alterations, EGFR gene amplification) that might have allowed relevant substratification of this subgroup. Our volume measurements were performed using FLAIR and T2WI, and we recognize that edema and gliosis might have been inadvertently included within measurements due to their overlapping appearance with infiltrative gliomas on these imaging sequences. Finally, we measured tumor volumes using MR imaging scans acquired on various scanners with variable image quality; such variables might have affected the volume measurements.

\section{CONCLUSIONS}

The extent of surgical resection differentially impacts overall survival among patients with LGGs based on molecular subtypes as defined by the WHO. Increased extent of surgical resection significantly benefits patients with IDH-mutant LGGs, with the impact strongest for IDHmut-Noncodel astrocytomas. We found no survival benefit from greater surgical resection in IDHwt LGGs. Further study of the IDHwt subgroup, with larger cohort sizes and molecular substratification, is warranted.

Disclosures: Sohil H. Patel—RELATED: Grant: Radiological Society of North American Research Scholar Grant (RSCH1819)*; UNRELATED: Expert Testimony: LeClairRyan Law Firm.* M. Beatriz Lopes-UNRELATED: Royalties: Elsevier, Comments: book chapter royalties; Travel/Accommodations/Meeting Expenses Unrelated to Activities Listed: International Society of Neuropathology, Comments: travel expenses to attend the international meeting in Tokyo, Japan, October 2018. Camilo E. FadulUNRELATED: Grants/Grants Pending: Novartis, Comments: clinical trial for recurrent glioblastoma. * Money paid to institution.

\section{REFERENCES}

1. Louis DN, Perry A, Reifenberger G, et al. The 2016 World Health Organization Classification of Tumors of the Central Nervous System: a summary. Acta Neuropathol 2016;131:803-20 CrossRef Medline

2. Yan H, Parsons DW, Jin G, et al. IDH1 and IDH2 mutations in gliomas. N Engl J Med 2009;360:765-73 CrossRef Medline

3. Brat DJ, Verhaak RG, Aldape KD, et al; Cancer Genome Atlas Research Network. Comprehensive, integrative genomic analysis of diffuse lower-grade gliomas. N Engl J Med 2015;372:2481-98 CrossRef Medline

4. Eckel-Passow JE, Lachance DH, Molinaro AM, et al. Glioma groups based on $1 \mathrm{p} / 19 \mathrm{q}, \mathrm{IDH}$, and TERT promoter mutations in tumors. N Engl J Med 2015;372:2499-508 CrossRef Medline

5. Ceccarelli M, Barthel FP, Malta TM, et al. Molecular profiling reveals biologically discrete subsets and pathways of progression in diffuse glioma. Cell 2016;164:550-63 CrossRef Medline
6. Cairncross G, Wang M, Shaw E, et al. Phase III trial of chemoradiotherapy for anaplastic oligodendroglioma: long-term results of RTOG 9402. J Clin Oncol 2013;31:337-43 CrossRef Medline

7. van den Bent MJ, Brandes AA, Taphoorn MJ, et al. Adjuvant procarbazine, lomustine, and vincristine chemotherapy in newly diagnosed anaplastic oligodendroglioma: long-term follow-up of EORTC brain tumor group study 26951. J Clin Oncol 2013;31:344-50 CrossRef Medline

8. Di Carlo DT, Duffau H, Cagnazzo F, et al. IDH wild-type WHO grade II diffuse low-grade gliomas: a heterogeneous family with different outcomes-systematic review and meta-analysis. Neurosurg Rev 2018 Jun 26. [Epub ahead of print] CrossRef Medline

9. Aibaidula A, Chan AK, Shi Z, et al. Adult IDH wild-type lower-grade gliomas should be further stratified. Neuro Oncol 2017;19:1327-37 CrossRef Medline

10. Poulen G, Goze C, Rigau V, et al. Huge heterogeneity in survival in a subset of adult patients with resected, wild-type isocitrate dehydrogenase status, WHO grade II astrocytomas. J Neurosurg $2018 \mathrm{Apr}$ 20:1-10. [Epub ahead of print] CrossRef Medline

11. Brat DJ, Aldape K, Colman H, et al. cIMPACT-NOW update 3: recommended diagnostic criteria for "diffuse astrocytic glioma, IDHwildtype, with molecular features of glioblastoma, WHO grade IV." Acta Neuropathol 2018;136:805-10 CrossRef Medline

12. Hervey-Jumper SL, Berger MS. Evidence for improving outcome through extent of resection. Neurosurg Clin N Am 2019;30:85-93 CrossRef Medline

13. Smith JS, Chang EF, Lamborn KR, et al. Role of extent of resection in the long-term outcome of low-grade hemispheric gliomas. J Clin Oncol 2008;26:1338-45 CrossRef Medline

14. Ius $\mathrm{T}$, Isola $\mathrm{M}$, Budai $\mathrm{R}$, et al. Low-grade glioma surgery in eloquent areas: volumetric analysis of extent of resection and its impact on overall survival-a single-institution experience in 190 patients: clinical article. J Neurosurg 2012;117:1039-52 CrossRef Medline

15. McGirt MJ, Chaichana KL, Attenello FJ, et al. Extent of surgical resection is independently associated with survival in patients with hemispheric infiltrating low-grade gliomas. Neurosurgery 2008;63: 700-07; author reply 707-08 CrossRef Medline

16. Roelz R, Strohmaier D, Jabbarli R, et al. Residual tumor volume as best outcome predictor in low grade glioma: a nine-years near-randomized survey of surgery vs. biopsy. Sci Rep 2016;6:32286 CrossRef Medline

17. Thon N, Kreth FW, Tonn JC. The role of surgery in grade II/III oligodendroglial tumors. CNS Oncol 2015;4:317-23 CrossRef Medline

18. Jakola AS, Myrmel KS, Kloster R, et al. Comparison of a strategy favoring early surgical resection vs a strategy favoring watchful waiting in low-grade gliomas. JAMA 2012;308:1881-88 CrossRef Medline

19. Duffau H, Lopes M, Arthuis F, et al. Contribution of intraoperative electrical stimulations in surgery of low grade gliomas: a comparative study between two series without (1985-96) and with (19962003) functional mapping in the same institution. J Neurol Neurosurg Psychiatry 2005;76:845-51 CrossRef Medline

20. Wijnenga MM, French PJ, Dubbink HJ, et al. The impact of surgery in molecularly defined low-grade glioma: an integrated clinical, radiological, and molecular analysis. Neuro Oncol 2018;20:103-12 CrossRef Medline

21. Eseonu CI, Eguia F, ReFaey K, et al. Comparative volumetric analysis of the extent of resection of molecularly and histologically distinct low grade gliomas and its role on survival. J Neurooncol 2017; 134:65-74 CrossRef Medline

22. Capper D, Weissert S, Balss J, et al. Characterization of $\mathbf{R} \mathbf{1 3 2} \mathbf{H}$ mutation-specific IDH1 antibody binding in brain tumors. Brain Pathol 2010;20:245-54 CrossRef Medline

23. Capper D, Zentgraf H, Balss J, et al. Monoclonal antibody specific for IDH1 R132H mutation. Acta Neuropathol 2009;118:599-601 CrossRef Medline

24. Felsberg J, Wolter M, Seul H, et al. Rapid and sensitive assessment of the IDH1 and IDH2 mutation status in cerebral gliomas based on DNA pyrosequencing. Acta Neuropathol 2010;119:501-07 CrossRef Medline 
25. Ahmadi R, Rezvan A, Dictus C, et al. Long-term outcome and survival of surgically treated supratentorial low-grade glioma in adult patients. Acta Neurochir (Wien) 2009;151:1359-65 CrossRef Medline

26. McGirt MJ, Chaichana KL, Gathinji M, et al. Independent association of extent of resection with survival in patients with malignant brain astrocytoma. J Neurosurg 2009;110:156-62 CrossRef Medline

27. Yeh SA, Ho JT, Lui CC, et al. Treatment outcomes and prognostic factors in patients with supratentorial low-grade gliomas. $\mathrm{Br} \mathrm{J} \mathrm{Ra}$ diol 2005;78:230-35 CrossRef Medline
28. Kawaguchi T, Sonoda Y, Shibahara I, et al. Impact of gross total resection in patients with WHO grade III glioma harboring the IDH $1 / 2$ mutation without the $1 \mathrm{p} / 19 \mathrm{q}$ co-deletion. J Neurooncol 2016;129:505-14 CrossRef Medline

29. Patel T, Bander ED, Venn RA, et al. The role of extent of resection in IDH1 wild-type or mutant low-grade gliomas. Neurosurgery 2018; 82:808-14 CrossRef Medline

30. van den Bent MJ. Interobserver variation of the histopathological diagnosis in clinical trials on glioma: a clinician's perspective. Acta Neuropathol 2010;120:297-304 CrossRef Medline 